Instituto Internacional de Investigación y Desarrollo Tecnológico Educativo INDTEC, C.A.

DOI: https://doi.org/10.29394/scientific.issn.2542-2987.2017.2.5.13.242-261

OAI-PMH: http://www.indteca.com/ojs/index.php/Revista Scientific/oai

\title{
Análisis Prospectivo de la Gerencia de Enfermería desde las Voces de los Estudiantes
}

Autor: Rafael Alejandro Camejo Giménez Universidad Centroccidental "Lisandro Alvarado", UCLA rafael.camejo@ucla.edu.ve Lara, Venezuela

\section{Resumen}

Este artículo científico tuvo como intención el estudiar la gerencia en la enfermería desde un análisis prospectivo, interpretar los sentidos y significados que le otorgan los actores sociales al futuro de la gerencia de enfermería y develar la importancia de una gerencia en enfermería desde la prospectividad. El paradigma fue interpretativo bajo un enfoque cualitativo, donde se aplicó el método fenomenológico apoyado en la hermenéutica. Se seleccionaron 05 estudiantes del primer semestre de enfermería y 05 del octavo para un total de 10 actores sociales. Como técnica para obtener la información utilicé como recursos la entrevista en profundidad, notas, fotografías y grabaciones. Emergieron de los hallazgos las siguientes categorías: Gerencia de servicio: formación, administración y funciones; Prospectividad: perfil y escenario futurista. Se triangularon las categorías emergentes y puedo decir que todo bachiller que incursione en la carrera debe conocer las funciones de enfermería. Se consideró el aporte del teórico Michel Godet, entre otros. Finalmente, como hallazgo principal se conoció que la mayoría de los estudiantes de enfermería no conocen el futuro de la carrera y la UCLA en el Decanato en estudio no aplica la gerencia prospectiva para determinar indicadores cognitivos y mentales en los futuros egresados de Enfermería.

Palabras clave: gerencia; prospectiva; enfermería. 


\title{
Prospective Analysis of Nursing Management from the Students Voices
}

\begin{abstract}
This scientific article was intended to study management in nursing from a prospective analysis, interpret the meanings and meanings that social actors grant to the future of nursing management and unveil the importance of nursing management from prospectivity. The paradigm was interpretative under a qualitative approach, where the phenomenological method based on hermeneutics was applied. We selected 05 students from the first semester of nursing and 05 from the eighth for a total of 10 social actors. As a technique for obtaining the information I used as resources the in-depth interview, notes, photographs and recordings. The following categories emerged from the findings: Service management: training, administration and functions; Prospectivity: profile and futuristic scenario. The emerging categories triangulated and I can say that every bachelor who enters the career must know the functions of nursing. The contribution of the theoretician Michel Godet, among others, was considered. Finally, as a main finding, it was known that most nursing students do not know the future of the career and UCLA in the Deanery under study does not apply prospective management to determine cognitive and mental indicators in future Nursing graduates.
\end{abstract}

Keywords: management; prospective; nursing. 


\section{Introducción}

Los estudios sobre predicción del éxito académico y profesional llevado a cabo en las universidades han tenido como soporte fundamental la gerencia desde una visión tradicional lo cual ha hecho que la visión de futuro se limite, bien sea a corto plazo, en la planificación de semestres, en el cumplimiento de actividades, en la búsqueda de recursos sin control, entre otros y en el mejor de los casos a mediano plazo ocasionando estancamiento en el desarrollo curricular de algunas materias como la administración de enfermería.

Asimismo para los efectos de la gerencia prospectiva, resulta importante mencionar situaciones de conflicto y de los procesos de negociación vividas en las universidades públicas, como es el caso de la UCLA, son situaciones emergentes que inciden de una $u$ otra forma en el ejercicio gerencial del enfermero, sus competencias e incluso sus aptitudes gerenciales necesarias, cada vez más, se asocian a la integración con credibilidad, respeto, trabajo en equipo y desarrollo de prácticas comprometidas con el cuidado integral del ser humano.

Es por ello que la gerencia en enfermería, bajo una visión prospectiva debe ser de naturaleza teórico - práctica y ser consecuente a la formación profesional de manera permanente, por cuanto contiene la administración, dirección y liderazgo organizacional, al permitir que el profesional que egresa de la universidad posea las habilidades y destrezas en su desarrollo profesional y pueda administrar los cuidados de enfermería de manera eficiente y eficaz, logrando desenvolverse como gerente y líder de excelencia.

\section{Configuración de la Realidad}

Las organizaciones a escala mundial, están adoptando nuevas técnicas, herramientas y métodos gerenciales para alcanzar ventajas competitivas y mejorar la calidad del producto o servicio, de tal forma de mantenerse en el mercado acorde a las exigencias internas y externas del 
ámbito donde se desenvuelven y así seguir satisfaciendo los requerimientos de los clientes.

Es importante mencionar que según Godet (2000), el estudio de la prospectividad puede realizarse en varios métodos entre ellos el escenario, el cual es "un conjunto formado por la descripción de una situación futura y la progresión de los acontecimientos que permiten pasar de la situación de origen a la situación de futuro". (pág. 17). Asimismo, explica que existen dos grandes tipos de escenarios: el exploratorio, que partiendo de las tendencias pasadas y presentes, conducen a futuros verosímiles, y el de anticipación o normativos, que construidos a partir de imágenes alternativas del futuro, podrán ser deseables o por el contrario rechazables.

Se interpreta que, en la actualidad una disciplina encargada de los estudios del futuro conocida como prospectiva, cuyo propósito es definir escenarios estrechamente ligados al futuro que de diversas maneras identifiquen ciertas variables y actores involucrados en el proceso, sobre los cuales se deberá actuar para alcanzar el futuro deseado o de lo contrario, si no se cuenta con la capacidad y determinación para hacerlo no se obtendrán los resultados esperados, identificar aquellos eventos que nos permitan accionar para explotar las oportunidades, disminuir 0 eliminar las vulnerabilidades y transformar las amenazas que se presentan en oportunidades.

En la actualidad, los aspectos vinculados a la gerencia de enfermería han adquirido una especial importancia a nivel de la gestión del sistema educativo. Así mismo, la sociedad actual demanda un sujeto capaz de conocerse a sí mismo y de conocer que oportunidades laborales y académicas le son más dadas de acuerdo a sus aptitudes y actitudes que acompaña al individuo en su formación durante toda la vida, de manera que conozca y tome decisiones para construir su propio conocimiento de acuerdo a su futuro.

En este sentido, el conocimiento es patrimonio de cada individuo, la 
Educación Universitaria se convierte en un vehículo apropiado para lograr orientar a los jóvenes que ingresan a este nivel educativo adecuadamente y así poder cumplir los objetivos que se han trazado. Lo antes expuesto, ha sido motivo de estudios continuos en el sistema educativo venezolano específicamente en el nivel de Educación Universitaria en gran parte, según Valero y otros (2006), por los nuevos paradigmas como la gestión del conocimiento, las nuevas tecnologías de información y comunicación, los modelos de escenarios prospectivos, entre otros, transformando positivamente el proceso de enseñanza y aprendizaje, donde el docente universitario y el estudiante son los protagonistas.

Es por ello, que desde la perspectiva de lo estudiado por el autor, el análisis prospectivo en el ámbito educativo busca la articulación entre la Educación Universitaria y la transformación productiva y de servicio. En este contexto presentado, Nery (2010) explica que la gerencia en enfermería, busca dar la mejor atención y que el personal de menor jerarquía se sienta satisfecho de trabajar, con los recursos que proporciona la institución, para dar un mejor servicio al usuario con bases científicas y sus competencias. Se interpreta que la gerencia en enfermería es un proceso que implica la coordinación directa e indirecta de todos los recursos disponibles en una organización (humanos, físicos, tecnológicos, financieros), a través de los procesos de: planificación, organización, dirección y control para el logro de los objetivos planteados.

En relación a lo mencionado y considerando que la gerencia en enfermería debe ser vista con una visión futurista, ésta se convierte en un arte a través de la ciencia, que busca conducir al equipo de manera estratégica, multidisciplinaria y organizativa para brindar una atención de calidad, logrando que haya un equilibrio entre el paciente, los subordinados y la institución, revisando el pasado, considerando el presente, pero proyectando el futuro.

De acuerdo a las investigaciones realizadas y mi experiencia como investigador y docente de la carrera en la UCLA, he podido conocer que la 
gerencia en enfermería es aprendida de manera implícita a través de la formación académica en aula y su puesta en marcha en las áreas de atención. Asimismo, los gerentes en el área de enfermería se capacitan después de posicionarse en un cargo y no se proyectan hacia el futuro, sino que se concentran en su presente y los problemas que este tiene, por ello es necesario estudiar la gerencia en enfermería prospectiva, de tal manera de lograr una sistematización de la praxis diaria para poder facilitar procesos que contribuyan a desarrollar competencias.

Para poder profundizar sobre los planteamientos hechos anteriormente, el investigador considera pertinente realizar las siguientes interrogantes ¿Qué significado confieren los actores sociales a la gerencia en enfermería como herramienta proyectiva en la carrera de enfermería de la UCLA?, ¿Cómo se interpretan los sentidos y significados que le otorgan los actores sociales sobre la gerencia en enfermería desde la prospectividad.?, ¿Cuál es la importancia de una gerencia en enfermería desde la prospectiva que modele indicadores para el mejoramiento de la carrera?.

\subsection{Intencionalidades}

\subsubsection{Intencionalidad General}

Estudiar la gerencia en enfermería como herramienta proyectiva en la carrera de enfermería de la UCLA.

\subsubsection{Intencionalidad Específica}

Interpretar los significados y sentidos que le otorgan los actores sociales sobre la gerencia en enfermería desde la prospectividad.

Develar la importancia de una gerencia en enfermería desde la prospectiva que modele indicadores para el mejoramiento de la carrera. 


\section{Sustentos Teóricos}

En este momento de la investigación se buscó compilar estudios, posiciones y postulados teóricos que me permitieron articular una visión compartida sobre la gerencia en enfermería como herramienta proyectiva en la carrera de enfermería de la UCLA. De allí que la prospectiva de acuerdo a Bass (1999) es también conocida como futurología y es:

Una disciplina sistemática que estudia el futuro desde una perspectiva, científica y tecnológica con la intención de comprenderlo y de poder influir en él... viene a ser el conjunto de tentativas sistemáticas para observar a largo plazo el futuro de la ciencia, la tecnología, la economía y la sociedad con el propósito de identificar las tecnologías emergentes que probablemente produzcan los mayores beneficios económicos o sociales. (pág. 82).

Se puede deducir del concepto presentado que el futuro es susceptible de ser creado y modificado por nosotros, para lo cual se tiene que tener una actitud proactiva hacia el cambio y generar los escenarios futuros más deseables. Por su parte, Miklos y Tello (2005), explican que la prospectiva es:

Una disciplina con visión global, sistémica, dinámica y abierta que explica los posibles futuros, no sólo por los datos del pasado sino fundamentalmente teniendo en cuenta las evoluciones futuras de las variables (cuantitativas y sobre todo cualitativas) así como los comportamientos de los actores implicados, de manera que reduce la incertidumbre, ilumina la acción presente y aporta mecanismos que conducen al futuro aceptable, conveniente o deseado. (s/p).

La prospectiva es una disciplina y un conjunto de metodologías orientadas a la previsión del futuro donde básicamente se trata de imaginar escenarios futuros posibles, denominados futuribles, y en ocasiones de determinar su probabilidad, con el fin último de planificar las acciones necesarias para evitar o acelerar su ocurrencia. Desde tal perspectiva, la prospectiva es entendida como una sistemática mental que, en su tramo más 
importante, viene desde el futuro hacia el presente, para finalmente concebir estrategias de acción tendientes a alcanzar el futuro objetivado como deseable, tal como lo señalan Miklos y Tello (ob.cit.):

Entre los propósitos importantes de esta aproximación cabe mencionar los siguientes: (a) Generar visiones alternativas de futuros deseados. (b) Proporcionar impulsos para la acción. (c) Promover in formación relevante bajo un enfoque de largo alcance. (d) Hacer explícitos escenarios alternativos de futuros posibles. (e) Establecer valores y reglas de decisión para alcanzar el mejor futuro posible. (pág. 42).

Se interpreta de lo presentado, que la prospectiva mantiene un amplio horizonte temporal, donde el interés es por eventos y situaciones a largo plazo, lo que trae consigo la flexibilidad ya que, por tratarse de una visión a alcanzar en varios años, permite la elección de futuros alternativos.

\subsection{La Prospectiva y su Relación con la Educación.}

La sociedad contemporánea está viviendo un ritmo que cada día es mucho más cambiante. De acuerdo a Bas (ob.cit.), las instituciones educativas de nivel superior necesitan tomar decisiones importantes y que trascienden el corto plazo; así todas aquellas relacionadas con el capital, inversiones en activos fijos, adquisición de tecnología, estrategias de mercados, son suficientemente importantes por los recursos a implicar como para que no se modifiquen sustancialmente transcurrido poco tiempo desde su adopción. Es evidente que en el ámbito educativo el concepto de prospectiva se mueve en marcos de competencia cada vez mayores, salvo algunas excepciones de carácter oligopolístico a quienes se les establecen límites cada vez más restringidos para que se abran a la competencia.

\subsection{Estrategia Gerencial en Educación desde la Prospectividad.}

La gerencia educativa prospectiva, constituye una metodología 
orientada a la previsión del futuro para determinar su probabilidad en escenarios futuros con el fin último de planificar las acciones necesarias para acelerar su ocurrencia. Plantea Godet (ob.cit.) que las herramientas que se utilizan en la prospectiva permiten organizar y estructurar de manera transparente y eficaz la reflexión colectiva sobre las apuestas y retos de futuro, además de la evaluación de las opciones estratégicas. Explica, que las estrategias en función de los escenarios pueden ser: (a) $\mathrm{Si}$ el grado de incertidumbre no es alto, se podría optar; bien por una estrategia arriesgada (haciendo las apuestas sobre un escenario situado entre los más probables).

(b) Si la incertidumbre fuese elevada convendría adoptar una estrategia flexible que abarque el máximo de opciones estratégicas reversibles.

El diseño del futuro se logra necesariamente a través de la redacción de escenarios, se caracterizan por facilitar la construcción del futuro, tarea en la cual intervienen factores de diferente índole. La universidad es una organización social, pues en ella no sólo se toman decisiones con respecto al proceso de enseñanza-aprendizaje; es además un espacio donde coexisten y se generan procesos para la coordinación y administración de recursos financieros, humanos y del conocimiento. La preocupación por el tema de la gestión, la calidad, la evaluación y el cambio en estas instituciones ha sido abordada por distintos autores y organismos internacionales desde distintas perspectivas.

\section{Metodología}

Este estudio se ubica en una investigación de carácter cualitativo focalizada en el paradigma interpretativo, el cual según Martínez (2009), tiene de naturaleza un perfil dialectico y sistémico que viene a resumir presupuestos epistemológicos y ontológicos en un corpus teórico-metodológico. Por tanto, la postura epistémica que consideré es apropiada para estudiar, comprender e interpretar como piensa y reflexiona el ser humano cuya responsabilidad es 
prepararse para ser enfermero (a) egresado de la UCLA. Observo que al considerar el conocimiento debo hacerlo bajo el entendimiento que éste es un producto y a la vez un proceso dentro de la interacción dialéctica que se da entre lo cognoscente y lo cognoscible.

Con respecto al método utilizado, en correspondencia a la intencionalidad y al fenómeno de estudio, asumí el fenomenológicohermenéutico, el cual es definido por Martínez (ob.cit.), como "el estudio de los fenómenos tal cual como son experimentados, vividos y percibidos por el hombre" (pág. 167). Expresa el autor que este el método más apropiado cuando la intención es:

...las realidades cuya naturaleza y estructura peculiar sólo pueden ser captadas desde el marco de referencia interno del sujeto interno que las vive y experimenta, exigen ser estudiadas mediante el método fenomenológico. En este caso no se está estudiando "una realidad objetiva y externa" (como ordinariamente se califica), igual para todos, sino una realidad cuya esencia depende del modo en que es vivida y percibida por el sujeto, una realidad interna y personal, única y propia de cada ser humano (pág. 167).

En este sentido, interpreto que la cualidad emergente se refiere a los cambios que se producen en el proceso investigativo para adaptarse a la pluralidad de opiniones de los actores sociales consultados, a su entorno y complejidad de tal manera de facilitar el flujo de las construcciones, versiones y significados facilitando su interpretación para dar sentido a situaciones, relacionando lo observado y escuchado. La fenomenología es definida por Hegel (citado por Hurtado, 2007), como "ciencia de las experiencias de la conciencia" (pág. 105). De este modo el fin de la fenomenología según Husserl, citado por Martínez (ob.cit.), "no es tanto describir un fenómeno singular, sino descubrir en él la esencia válida universalmente y útil científicamente". (pág. 139).

En otras palabras, el método seleccionado, de acuerdo a Martínez 
(ob.cit.) permite el estudio de las realidades, cuya naturaleza y estructura peculiar sólo pueden ser captadas dentro del marco de referencia interno del sujeto que la vive y experimenta. De igual manera, Ray (2009), destaca que la fenomenología tiene que ver con la pregunta ¿cómo conocemos?, tal como lo refleja la actitud que asume el padre de la fenomenología Husserl (1970), al manifestar que podría ser utilizada como una filosofía, un enfoque y un método.

Con respecto al método hermenéutico, según Martínez (ob.cit.) "tiene como misión descubrir los significados de las cosas, interpretar lo mejor posible las palabras, los escritos, los textos y los gestos, así como cualquier acto u obra, pero conservando su singularidad en el contexto de que forma parte" (pág. 119). Es importante, señalar que con la hermenéutica, me acercaré a develar los fenómenos ocultos y, en particular sus significados. Para lograrlo admití la rigurosidad necesaria a través de las siguientes fases: (a) comprensión, porque me apoyé en construcciones - proyectos, elaborados a partir de fuentes originarias, anticipados por el hermeneuta y constatadas en el mundo de la vida; (b) interpretación, donde integre lo aspirado por el intérprete y lo ofrecido significativamente por el texto el acto humano; (c) aplicación, donde validé los enunciados interpretados al dibujar nuevos horizontes desde lo ya construido hacia lo construible.

\subsection{Técnicas de Recolección}

Como técnica para obtener la información utilice la entrevista en profundidad, que según refieren Taylor y Bogdan (2002), consiste en reiterados encuentros cara a cara con cada informante, dirigidos hacia la comprensión de las perspectivas que tienen respecto a sus vidas, experiencias o situaciones tal como lo expresan con sus propias palabras. Por consiguiente, es imprescindible realizarlas en un ambiente relajado y con tranquilidad, lo que permitirá la fluidez de la información en el modo en que ellos ven y conciben 
su realidad. Por ello, solicitaré su autorización para tomar notas, fotografías y grabar, a fin de tener registros y cumplir con los principios de credibilidad y fiabilidad de la investigación.

Paso seguido triangulé las categorías emergentes, con la finalidad de garantizar la calidad del proceso investigativo, por lo cual me apoyé en ella, para presentar como síntesis del proceso hermenéutico desarrollado en cada categoría, un cuadro donde se muestra la triangulación de estas, indicando cuales surgieron de cada informante. Luego presenté la triangulación de fuentes, donde se ilustran las categorías coincidentes entre los versionantes, e incluso aquellas emergentes entre dos versionantes, siendo estas relevantes y por ende, aportan para la investigación, por cuanto las considero proporciona credibilidad a los resultados. La triangulación me permitió aportar elementos a la credibilidad de la información, acentuándose los puntos concurrentes emergentes de la dialéctica de todos los actores sociales. De los hallazgos obtenidos emergieron las siguientes unidades de análisis y categorías, como se muestra en el cuadro $N^{\circ} 01$ :

Cuadro 1. Unidades, Categorías y Dimensiones.

\begin{tabular}{|c|c|c|}
\hline Unidad de Análisis & Categoría de Análisis & Dimensión \\
\hline \multirow{3}{*}{$\begin{array}{l}\text { Gerencia en } \\
\text { Enfermería }\end{array}$} & Formación & $\begin{array}{l}\text { - Conocimiento } \\
\text { - Técnicas } \\
\text { - Estructura }\end{array}$ \\
\hline & Administración & $\begin{array}{l}\text { - Planificación } \\
\text { - Organización } \\
\text { - Dirección } \\
\text { - Control }\end{array}$ \\
\hline & Funciones & $\begin{array}{l}\text { - Asistencial } \\
\text { - Educativo } \\
\text { - Gerencial } \\
\text { - Investigativo }\end{array}$ \\
\hline Prospectividad & Perfil & $\begin{array}{l}\text { - Liderazgo } \\
\text { - Trabajo en Equipo } \\
\text { - Administración }\end{array}$ \\
\hline
\end{tabular}




\begin{tabular}{|l|l|l|}
\hline & & $\bullet$ Compromiso \\
\cline { 2 - 3 } & Escenario Futurista & $\begin{array}{l}\text { - Emprendedurismo } \\
\text { - Enfoque al paciente } \\
\text { - Negociación }\end{array}$ \\
\hline
\end{tabular}

Fuente: El Autor (2017).

Una vez elaborado la matriz de resumen sobre el proceso categorial, se procedió a mostrar las áreas de discusión, categorías, dimensiones y colores que identifican categorías y dimensiones, se triangularon las categorías emergentes, y puedo decir que todo bachiller que incursione en la carrera de enfermería debe estar claro que sus funciones son asistenciales, gerenciales, docentes e investigativas, desde el contexto específico de cuidado, en los niveles primarios, secundario y terciario, con fundamento en un real conocimiento del ser humano, de la salud y sus determinantes, y del entorno. Seguidamente se muestra la triangulación de las categorías emergentes en el cuadro $\mathrm{N}^{\circ} 2$ :

Cuadro 2. Triangulación de las Categorías Emergentes.

\begin{tabular}{|c|c|c|c|c|c|c|c|}
\hline Unidad de Análisis & $\begin{array}{c}\text { Categoría } \\
\text { Emergente }\end{array}$ & IC 1 & IC 2 & IC 3 & IC 4 & IC 5 & IC 6 \\
\hline \multirow{2}{*}{$\begin{array}{c}\text { Gerencia en } \\
\text { Enfermería }\end{array}$} & Formación & & & & & & \\
\hline & Administración & & & & & & \\
\hline & Funciones & & & & & & \\
\hline \multirow{4}{*}{ Prospectividad } & Perfil & & & & & & \\
\hline & $\begin{array}{c}\text { Escenario } \\
\text { Futurista }\end{array}$ & & & & & & \\
\hline
\end{tabular}

Fuente: El Autor (2017).

Partiendo de los hallazgos, es necesario abordar la teorización en la investigación; así Martínez (2009), explica que el fin de la teorización (y de la categorización que le precede) “...es lograr estructurar una imagen 
representativa, un patrón coherente lógico, un modelo teórico o una auténtica teoría o configuración del fenómeno estudiado, que le dé sentido a todas sus partes y componentes..." (pág. 56). Para este autor, posterior al proceso de categorización, se puede lograr alcanzar una teoría en la cual el investigador hace emerger desde las voces extraídas de los actores sociales un modelo teórico, un aporte, que le dé sentido y aporte al proceso investigativo.

Es así como considero que es el conjunto de categorías que me permite observar a largo plazo el futuro de la carrera de enfermería, considerando la formación vista desde el conocimiento adquirido y por adquirir. Me permito significar que no se busca la articulación entre la educación universitaria y la transformación productiva y debe ser concebido el desarrollo para la formación de los recursos humanos para introducir y difundir los lineamientos doctrinarios y teóricos basado en nuevos paradigmas de transformación administrativa, tecnológico, operativa y gerencial suministrando un nuevo entendimiento sobre la gerencia de servicio, de tal forma de egresar estudiantes con perfiles académicos basados en liderazgo, trabajo en equipo, administración y compromiso.

\subsection{Actores Sociales}

Reflexionando sobre lo expresado por los autores citados, articule lo interpretado del fenómeno estudiado según los actores sociales que participaron en el proceso investigativo, con una visión humanista, abierta, rigurosa. De allí que, se seleccionan los estudiantes de primer semestre de enfermería considerando 05 actores sociales y 05 del octavo semestre para un total de 10 actores sociales. Como técnica para obtener la información utilicé la entrevista en profundidad. De igual manera, solicite autorización a los actores sociales para tomar notas, fotografías y grabar, con el fin de tener registros para de esta manera cumplir con los principios de credibilidad y fiabilidad de la investigación. 


\subsection{Categorización de la Información}

Esta categorización se realizó luego de haber recopilado la información de los actores sociales y considerando la experiencia del investigador que como, inicio la etapa de estructuración de los datos, lo que implicó organizar las unidades, para la categorización y sacar lo mejor de cada una de las expresiones, significantes y significados de las personas entrevistadas le otorgan a su experiencia.

En este sentido, Martínez (ob. cit.) afirma que las categorías surgen de lo estudiado, en tal sentido, categorizar "consiste en clasificar, conceptuar o codificar mediante un término o expresión que sea claro e inequívoco (categoría descriptiva) el contenido o idea central de cada unidad temática" ( $p$. 75). A tales efectos, use matrices para organizar la información, considerando a efectos de identificar las categorías la técnica de cromatización. Igualmente utilice un primer conjunto de matrices a efectos de categorizar la información, en las cuales colocare las categorías, dimensiones, número para identificar las líneas y el texto.

Posteriormente agrupé en otro juego de matrices por versionante la información. Para el análisis de contenido utilice los textos resultantes de las entrevistas, lo cual permitirán precisar las categorías que ayudarán a la interpretación y teorización de los resultados. En tal sentido, la información que se obtenga quedará conformada por dimensiones de análisis, las cuales no se preestablecen, emergen de la realidad.

\subsection{Fiabilidad y Credibilidad de la Investigación}

Para Martínez (ob.cit.), la fiabilidad está orientada hacia el nivel de concordancia hacia diferentes, observadores, evaluadores o jueces del mismo fenómeno; de allí que se tomará en cuenta estos aspectos para estudiar la fiabilidad de la investigación. Según Pérez (2008), uno de los aspectos más controvertidos en la investigación cualitativa es el relacionado con los criterios 
de veracidad.

En los estudios cualitativos es necesario, al igual que en los cuantitativos, explicar, la validez y la fiabilidad de los resultados. Según Pérez (ob.cit.), lo que hace a una investigación cualitativa científica, es la observación prolongada, las entrevistas a profundidad, la triangulación de las percepciones e interpretaciones de los participantes y, en especial, la presencia crítica del observador en el contexto donde ocurre el fenómeno.

En virtud de que el término confiable no encaja dentro de la investigación cualitativa, Lincoln y Guba (2009), sugieren que se piense en la consistencia de los resultados obtenidos de la información. De este modo, la fiabilidad externa de este estudio se fundamentará, en la identificación de los supuestos o teorías en la que subyace el proceso, la descripción del contexto en que se recaban los datos y de los versionantes.

El realizar la triangulación, la cual es una herramienta que por su rigurosidad, me permitió determinar la consistencia de los resultados dándole credibilidad y fiabilidad a esta investigación, esta técnica es ampliada en la interpretación de los resultados con el uso de las matrices ya descritas, reforzando así la calidad de la investigación, así como de la teoría que se generará sobre la base de los hallazgos.

\section{Hallazgos}

Un primer hallazgo se fundamenta en que la mayoría de los estudiantes de enfermería no conocen el futuro de la carrera y alegan estancamiento en el mejoramiento profesional por falta de una gerencia adecuada al servicio del cliente interno (estudiantes y docentes) y externos (directiva de la carrera en la UCLA). Se conoció que la UCLA en el Decanato en estudio no aplica la gerencia prospectiva para determinar indicadores cognitivos y mentales en los futuros egresados de la carrera.

Asimismo, La mayoría de los futuros egresados de enfermería carecen 
de conocimiento sobre gerencia, es decir, carecen de preparación administrativa. Existe un nivel alto de aceptación del trabajo ejecutado, sin embargo, su debilidad es la falta de estudios que proyecten la administración de enfermería en el tiempo, tanto a corto, mediano como a largo plazo. La mayoría del personal de enfermería, se deben capacitar por su cuenta en el área gerencial lo que limita proyección de una gerencia eficaz y eficiente.

Otro hallazgo relevante está relacionado con el personal que imparte la administración de enfermería, el cual debe cumplir durante todo su ejercicio las siguientes funciones:

Asistencial: Con un adecuado fundamente científico, humano y ético.

Educativo: En el ámbito institucional y ambulatorio tanto para el individuo, la familia como la comunidad, brindar modelos de enseñanza basados en el autocuidado, igualmente actualizar el personal a su cargo o de formar personal de enfermería con carácter Profesional o Vocacional.

Gerencial: con liderazgo y compromiso, enfocado a la búsqueda de la calidad en la atención, al logro de los objetivos de la organización donde se desenvuelve y al desarrollo humano de su grupo de trabajo.

Investigativo: En construcción del conocimiento en salud para el desarrollo científico y técnico del sector, de las instituciones de salud y la Profesión de Enfermería.

Finalmente, no hay pertinencia de la enseñanza universitaria con la gerencia prospectiva por lo que no existen escenarios futuros ya que no se considera la sistematización del ejercicio profesional y los criterios de formación no se basan en estadísticas ni en proyecciones pues están formados de manera tradicional. 


\section{Reflexiones de Cierre}

La administración a nivel gerencial, implica una estructuración de las relaciones que debe existir entre funciones, niveles y actividades de los elementos materiales y humanos, con el fin de lograr la máxima eficiencia dentro de los planes y objetivos de una organización donde la coordinación entre todos estos elementos es una verdadera necesidad.

Así mismo, deben dirigirse las actividades de asistencia hacia el cumplimiento de metas y objetivos, pues proporciona armonía y comunicación entre los actos de las personas y las unidades administrativas de un organismo, facilitando el funcionamiento y cumplimiento exitoso de las actividades profesionales, para el caso del profesional de enfermería.

Es por ello que en la actualidad se deberá construir un nuevo modelo de gerencia en enfermería bajo el escenario prospectivo mediante el diseño e implantación de nuevos modelos curriculares (a nivel de la formación de futuros profesionales de enfermería) y modelos de escenarios prospectivos (para los profesionales en ejercicio), con atención a las demandas que la sociedad plantea que impulse una adecuada sinergia entre conocimientos, capacidades y actitudes para dotar a las organizaciones de gerentes con capacidad emprendedora, responsabilidad, creatividad y flexibilidad en su práctica profesional y administrativa.

Finalmente se sugieren las siguientes líneas de acción: (1) Conocimiento de la situación de salud en el área de enfermería y su vinculación gerencial para el mejoramiento de la práctica en la resolución de situaciones complejas. (2) Promoción de una visión gerencial a largo plazo. (3) Logro de mayor efectividad en la administración de recursos. (4) Participación en la educación basada en gerencia y prospectividad para actualizar la praxis laboral. (5) Formación y supervisión del personal con visión de futuro. (6) Adquisición de mayores competencias que hagan más eficiente el trabajo de enfermería. (7) Mejorar el proceso de formación, haciendo más atrayente la 
carrera de enfermería. (8) Incrementar la matrícula en el nivel universitario, lograr un control sobre las escuelas de nivel técnico y universitario actualizando los currículos y los métodos de enseñanza.

\section{Referencias}

Bass E. (1999). Prospectiva: Herramientas para la Gestión Estratégica del Cambio. Editorial Ariel. Barcelona.

Hurtado de B. J. (2007). El proyecto de investigación. Metodología de investigación holística. (2da. Ed.). Caracas: Fundación Sypal.

Husserl, E. (1970). A Ideia da Fenomenología. Trad. ArturMorão. Lisboa, Port.: Edições 70, 2014.

Lincoln y Guba. (2009). Naturalisticinquiry. E.U.A.: California Sage Publication, Newbury Park.

Martínez, M. (2009). Comportamiento humano: Nuevos métodos de investigación. México: Trillas

Miklos, T. y Tello, M. (2005). Planeación Prospectiva. Una estrategia para el diseño del Futuro. México: Limusa.

Nery, A. (2010). Una aproximación al concepto de gerencia y administración aplicado a la disciplina de enfermería. Revista de Enfermagem 2010, № 14.

Pérez, G. (2008). Investigación cualitativa. Retos e interrogantes. Técnicas yanálisis de datos. Madrid: La Muralla.

Ray, M. (2009). La riqueza de la fenomenología: preocupaciones filosóficas, teóricas y metodológicas. En Morse, J.M.: asuntos críticos en los métodos de investigación cualitativa. Colombia: Editorial Universidad de Antioquía

Taylor, S.J. y Bogdan, R. (2002). Introducción a los métodos cualitativos de investigación: la búsqueda de los significados. Madrid: Paidós. 


\section{Rafael Alejandro Camejo Giménez \\ e-mail: rafael.camejo@ucla.edu.ve}

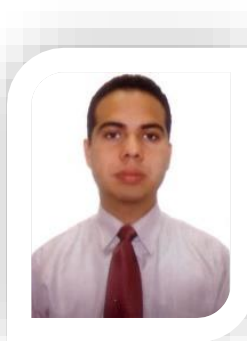

Nacido en Venezuela. Es egresado de la Universidad de los Andes - Mérida como Licenciado en Enfermería.

Cursa sus estudios Doctorales en Gerencia Avanzada en la Universidad Fermín Toro - Cabudare. Posee estudios de Post grado en Maestría en Educación Superior de la UPEL - IPB. Se ha desempeñado como Coordinador de Enfermería en diversos centros asistenciales. Actualmente es Docente Ordinario del Departamento de Enfermería de la UCLA y Coordinador de la Comisión de Investigación del Departamento. Es Jurado de Trabajos de Investigación de Pregrado en Enfermería y Coordinador de Actos de Grado del Decanato de Ciencias de la Salud de la UCLA.

El contenido de este manuscrito se difunde bajo una Licencia de Creative Commons ReconocimientoNoComercial-Compartirlgual 4.0 Internacional 